\title{
Application of Markov Model in Prediction of Annual Premium of Automobile
}

\author{
Yayun Fan, Jingjing Feng \\ Intelligent Science and Information Engineering College \\ Xi'an Peihua University, Xi'an, China \\ ziyun50051@163.com, fengjingjing0105@q163.com
}

Keywords:Markov model; Auto insurance; Annual insurance premium; Transfer matrix

\begin{abstract}
This paper establishes the Markov model based on a insurance system which ascending or descending the auto insurance premiums according to the former multi-state auto insurance. It assumes that the number of automobile claims is a Poisson random variable with parameter $\lambda$, then establishes a state transition matrix, finally forecasting the annual Insurance cost of automobiles by the steady distribution of transfer matrix. The results show that when the average number of annual claims of the insured person is $1 / 3$, the insurance premium will increase to the second state in the next year, and the annual insurance premium will increase to the first year when the average number of claims is 1,2 , and 3, In 3 states, when the number of claims exceeds 3 , it will increase to the 4th state. The prediction results provide data support for the car insurer, pay attention to the number of claims for annual claims, rationally plan funds, and maximize the benefits as much as possible.
\end{abstract}

\section{INTRODUCTION}

Since the reform and opening up, along with the economic and social development, china has accelerated its entry into the "dwell time", car ownerships are rising, as an important means of transport, vehicle accidents such as rollover, collision, scratch and other accidents are increasing, and therefore the business of insurance and claims increased. Vehicle insurance has received more and more attention from all walks of life, auto insurance claims have become an important issue. Insurance claim refers to the loss of insurance car in the scope of insurance liability of insurance, the underwriter depends on the agreement of insurance contract to solve the insurance compensation problem procedure. Claim work is an important embodiment of insurance policy and function, it is the underwriter carries out the insurance contract, carries out the insurance obligation, assumes the insurance responsibility concrete embodiment, the superiority of insurance and insurance given by the applicant economic compensation to a large extent by claim work. For car ginseng protect people, how to deal with to become a beneficiary to a great extent this is the main problem[1-3]. The Markov prediction model[4] is widely used in queuing theory[5], commodity sales[6], weather forecast[7], and disease infection[8]. However, there are very few researches on the annual insurance premiums that people are concerned about. This paper uses the Markov chain to forecast the average annual insurance premiums by car insurers based on the multi-state ascendingdescending auto-insurance systems. providing data support for car insurers, paying attention to annual claims for reasonable planning for the use of funds.

\section{II . MAIN METHODS}

Markov model is a probability model, Markov prediction is a modern prediction method. It has high science, accuracy and applicability, and is widely applied in natural science and economic management. The Markov prediction model takes time series as a process. It determines the trend of state changed predicts the future of things by studying the initial probability of different states and the probability of transition between states.

\section{A. Basic Definition}

Random process $\left\{X_{n}, n=0,1,2, \cdots\right\}$;

Parameter $T=\{0,1,2 \cdots\}$; 
State space $S=\left\{S_{0}, S_{1}, S_{2}, \cdots\right\}$.

If random process $\left\{X_{n}, n \in T\right\}$ for arbitrary integer $n \in T$ and $S_{0}, S_{1}, \cdots S_{n+1} \in S$, conditional probability satisfies: $\quad P\left\{X_{n+1}=i_{n+1} \mid X_{0}=i_{0}, X_{1}=i_{1}, \cdots X_{n}=i_{n}\right\}=P\left\{X_{n+1}=i_{n+1} \mid X_{n}=i_{n}\right\}$

then $\left\{X_{n}, n \in T\right\}$ is called the Markov chain.

\section{B. Conditions of Use}

The number of states of the system will not change; and the transition probability matrix will not change periodically; given the state of the past $S_{1}, S_{2}, \cdots, S_{n-1}$ and the state of the present $S_{n}$, the conditional distribution of the future state $S_{n+1}$ is independent of the state of the past and depends only on the present state.

\section{Transfer Probability Matrix Model}

The probability of state transfer refers to the possibility that in the course of the development of an event, from a certain state, the next moment is transferred to another state. If the system state changes, the number of states is $m$, that is $S_{1}, S_{2}, \cdots S_{m}$. The system is now in the $S_{i}$ states, the probability of the next step to the $S_{j}$ state is recorded as $P_{i j}$, and the total transfer of the system state can be represented by the following transfer matrix:

$$
P_{i j}=\left[\begin{array}{cccc}
p_{11} & p_{11} & \cdots & p_{1 m} \\
p_{21} & p_{21} & \cdots & p_{2 m} \\
\vdots & \vdots & \vdots & \vdots \\
p_{m 1} & p_{m 2} & \cdots & p_{m m}
\end{array}\right]
$$

And transfer matrix satisfaction:

$$
\sum_{j=1}^{m} p_{i j}=p_{i 1}+p_{i 2}+\cdots+p_{i m}=1 \quad(i=1,2, \cdots k)
$$

Depending on the state of the current moment and the state transition matrix, you can expect the following situation or the following period:

If the former state of the thing is, $S_{n-1}$ the state of the latter phase is $S_{n}$, and the state transfer matrix is $P$, then the relationship between the three is $S_{n}=S_{n-1} \cdot P$.

\section{III . ESTABLISH A MODEL}

Most of China's auto insurance premiums are determined by the ascending-descending system. Each insured person is given a positive integer value as a state, depend on the type of car and the level of insurance participation we can get a positive integer value as each car's annual insurance premiums. The status of the insured person changes with the number of insured claims, and the low status corresponds to a low annual premium. If the insured has no claims in the previous year, then his status will reduce, If the insured person has at least one claims claim in the previous year, his status will increase correspondingly. The more the number of claims is, the higher the annual premium in the next year will be. So no claims will lead to lower insurance premiums, requiring claims leads to higher insurance premiums, which is the insurance ascending-descending systems.

For an ascending-descending system given by an insurance company, $S_{i}(k)$ represents the next year's status of a car holder who has been in the $i$-state for the previous year and has $k$ claims or the year. Assuming the number of claims of a car insured person is a Poisson random variable with parameter $\lambda$, then the subsequent successor state of this vehicle insurer will form a Markov chain with a transition probability $P_{i, j}$,

$$
P_{i, j}=\sum_{k: S_{i}(k)=j} e^{-\lambda \frac{\lambda^{k}}{k !}} \quad j \geq 0
$$


At the same time, the probability that the insured person has $k$ claims in a year is the probability that $\alpha_{k}$,

$$
\alpha_{k}=e^{-\lambda \frac{\lambda^{k}}{k !}}, \quad k \geq 0
$$

Assume a four-state ascending-descending system, as shown in the following table:

TABLE I: INSURANCE STATE TRANSFER DIAGRAM

\begin{tabular}{|c|c|c|c|c|c|}
\hline \multirow{2}{*}{ status } & \multirow{2}{*}{ Annual insurance (yuan) } & \multicolumn{4}{|c|}{ Next state } \\
\cline { 2 - 6 } & & 0 claims & 1 claims & 2 claims & 3claims above \\
\hline 1 & Strong risk 900 & 1 & 2 & 3 & 4 \\
\hline 2 & Business insurance1 1500 & 1 & 3 & 4 & 4 \\
\hline 3 & Business insurance2 2500 & 2 & 4 & 4 & 4 \\
\hline 4 & Business insurance3 3500 & 3 & 4 & 4 & 4 \\
\hline
\end{tabular}

It can be seen from the table: $S_{2}(0)=1 ; S_{2}(1)=3$;

For the above table, there is a state transition matrix for the car insured person.

$$
P=\left[\begin{array}{cccc}
\alpha_{0} & \alpha_{1} & \alpha_{2} & 1-\alpha_{0}-\alpha_{1}-\alpha_{2} \\
\alpha_{0} & 0 & \alpha_{1} & 1-\alpha_{0}-\alpha_{1} \\
0 & \alpha_{0} & 0 & 1-\alpha_{0} \\
0 & 0 & \alpha_{0} & 1-\alpha_{0}
\end{array}\right]
$$

\section{CASE ANALYSIS}

Analyze a four-state ascending-descending car insurance system. As shown in the above table, we assume that the number of claims per year is a Poisson random variable whose mean values are $\lambda=1 / 3,1,2$, 3and 4 respectively. The average annual premium is $R$ paid by the insured person.

(1) $\lambda=1 / 3$, then, $\alpha_{k}=e^{-\lambda \frac{\lambda^{k}}{k !}}$,

get $\alpha_{0}=0.7165, \alpha_{1}=0.2388, \alpha_{2}=0.0398$,

The transfer matrix is

$$
P_{1}=\left[\begin{array}{cccc}
0.7165 & 0.2388 & 0.0398 & 0.0049 \\
0.7165 & 0 & 0.2388 & 0.0447 \\
0 & 0.7165 & 0 & 0.2835 \\
0 & 0 & 0.7165 & 0.2835
\end{array}\right]
$$

According to stationary probability and given 


$$
\left\{\begin{array}{c}
\pi_{11}=0.7165 \pi_{11}+0.7165 \pi_{12} \\
\pi_{12}=0.2388 \pi_{11}+0.7165 \pi_{13} \\
\pi_{13}=0.0398 \pi_{11}+0.2388 \pi_{12}+0.7165 \pi_{13} \\
\pi_{11}+\pi_{12}+\pi_{13}+\pi_{14}=1
\end{array}\right.
$$

The results of using mathematical are:

$$
\pi_{11}=0.5771, \pi_{12}=0.2283, \pi_{13}=0.1263, \pi_{14}=0.6819
$$

Similarly:

(2) $\lambda=1, \quad \alpha_{0}=0.3679, \alpha_{1}=0.3679, \alpha_{2}=0.1839$

(3) $\lambda=2, \quad \alpha_{0}=0.1353, \alpha_{1}=0.2707, \alpha_{2}=0.2707$

（4） $\lambda=3, \quad \alpha_{0}=0.0498, \alpha_{1}=0.1494, \alpha_{2}=0.2240$

(5) $\lambda=4, \quad \alpha_{0}=0.0183, \alpha_{1}=0.0733, \alpha_{2}=0.1465$

Then the transfer matrix is:

$$
\begin{aligned}
P_{2} & =\left[\begin{array}{cccc}
0.3679 & 0.3679 & 0.1839 & 0.0803 \\
0.3679 & 0 & 0.3679 & 0.2642 \\
0 & 0.3679 & 0 & 0.6321 \\
0 & 0 & 0.3679 & 0.6321
\end{array}\right] \\
P_{3} & =\left[\begin{array}{cccc}
0.1353 & 0.2707 & 0.2707 & 0.3233 \\
0.1353 & 0 & 0.2707 & 0.5940 \\
0 & 0.1353 & 0 & 0.8647 \\
0 & 0 & 0.1353 & 0.8647
\end{array}\right] \\
P_{4} & =\left[\begin{array}{cccc}
0.0498 & 0.1494 & 0.2240 & 0.5768 \\
0.0498 & 0 & 0.1494 & 0.8008 \\
0 & 0.0498 & 0 & 0.9502 \\
0 & 0 & 0.0498 & 0.9502
\end{array}\right] \\
P_{5} & =\left[\begin{array}{cccc}
0.0183 & 0.0733 & 0.1465 & 0.7536 \\
0.0183 & 0 & 0.0733 & 0.9084 \\
0 & 0.0183 & 0 & 0.9817 \\
0 & 0 & 0.0183 & 0.9817
\end{array}\right]
\end{aligned}
$$

According to stationary probability and given

$$
\begin{gathered}
\left\{\begin{array}{c}
\pi_{21}=0.3679 \pi_{21}+0.3679 \pi_{22} \\
\pi_{22}=0.3679 \pi_{21}+0.3679 \pi_{23} \\
\pi_{23}=0.1839 \pi_{21}+0.3679 \pi_{22}+0.3679 \pi_{23} \\
\pi_{21}+\pi_{22}+\pi_{23}+\pi_{24}=1
\end{array}\right. \\
\left\{\begin{array}{c}
\pi_{31}=0.1353 \pi_{31}+0.1353 \pi_{32} \\
\pi_{32}=0.2707 \pi_{31}+0.1353 \pi_{33} \\
\pi_{33}=0.2707 \pi_{31}+0.2707 \pi_{32}+0.1353 \pi_{33} \\
\pi_{31}+\pi_{32}+\pi_{33}+\pi_{34}=1
\end{array}\right.
\end{gathered}
$$




$$
\begin{aligned}
& \left\{\begin{array}{c}
\pi_{41}=0.0498 \pi_{41}+0.0498 \pi_{42} \\
\pi_{42}=0.1494 \pi_{41}+0.0498 \pi_{43} \\
\pi_{43}=0.2240 \pi_{41}+0.1494 \pi_{42}+0.0498 \pi_{43} \\
\pi_{41}+\pi_{42}+\pi_{43}+\pi_{44}=1
\end{array}\right. \\
& \left\{\begin{array}{c}
\pi_{51}=.0183 \pi_{51}+.0183 \pi_{52} \\
\pi_{52}=0.0733 \pi_{51}+.0183 \pi_{53} \\
\pi_{53}=0.1465 \pi_{51}+0.0733 \pi_{52}+.0183 \pi_{53} \\
\pi_{51}+\pi_{52}+\pi_{53}+\pi_{54}=1
\end{array}\right.
\end{aligned}
$$

Using mathematical the answers of the stationary probability are:

$$
\begin{aligned}
& \pi_{21}=0.0707, \pi_{22}=0.1215, \pi_{23}=0.2595, \pi_{24}=0.548 \\
& \pi_{31}=0, \pi_{32}=0, \pi_{33}=0.1191, \pi_{34}=0.880 \\
& \pi_{41}=0.0001, \pi_{42}=0.0024, \pi_{43}=0.0477, \pi_{44}=0.9498 \\
& \pi_{51}=0, \pi_{52}=0.0003, \pi_{53}=0.1799, \pi_{54}=0.9817
\end{aligned}
$$

From the above data, it can be seen that the number of annual claim claims of car insured person is a Poisson random variable whose mean value is $1 / 3,1,2,3$ and 4 , and the average annual insurance premium paid is $R$ : (rounded to retain the integer)

$$
\begin{aligned}
R(1) & =900 \times 0.5771+1500 \times 0.2283+2500 \times 0.1263+3500 \times 0.068 \\
& \approx 1416 \\
R(2) & =900 \times 0.0707+1500 \times 0.1215+2500 \times 0.2595+3500 \times 0.548 \\
& \approx 2814 \\
R(3) & =900 \times 0+1500 \times 0+2500 \times 0.1191+3500 \times 0.8808 \\
& \approx 3381 \\
R(4) & =900 \times 0.0001+1500 \times 0.0024+2500 \times 0.0477+3500 \times 0.9498 \\
& \approx 3448 \\
R(5) & =900 \times 0 .+1500 \times 0.0003+2500 \times 0.1799+3500 \times 0.9817 \\
& \approx 3886
\end{aligned}
$$

\section{CONCLUSIONS AND FUTURE WORK}

Based on the Markov model, the state of automobile insurance costs is calculated and analyzed for the annual insurance cost of automobiles. Then make an annual insurance expenses prediction analysis of the next state belonged to the car insured person with different number of claims .The results show that when the average number of annual claims of the insured person is $1 / 3$, the insurance premium will increase to the second status in the next year, and when the annual average number of claims is 1-3, the annual insurance premium will increase to the third state, From the results, it can be inferred that when the average number of claims in the current year is 4 or above, the annual premium for the next year will increase to the fourth state, and the theoretical results can enable the participants to make a better funds plan for the more reasonable utilization rate. At the same time, it should be noted that the more data is predicted, the more accurate it is when studying the model.

This article only states the four states of the insurance system. Actually, the insurance coverage is different, and the state is also more than four states. It is recommended that similar research projects be able to carry out more analysis of the status to guarantee forecast results are more accurate and provide stronger data support for car insured person. 


\section{ACKNOWLEDGMENT}

This work was funded by Xi'an Peihua University support project under the Grant No.PHKT17002. The authors would like to thank fund from department, as well as helpful opinions from colleagues.

\section{REFERENCES}

[1] J.J.Peng, Study on Risk Control of Vehicle Insurance based on Data Mining, Jilin university,Dec.2014.

[2] X.Y.Zhang, Talk about car insurance and claims, Wireless Internet Technology, pp.115,Jan.2014.

[3] H.Chen,T.T.Wei, Bibliometric analysis of the research hot spots of vehicle insurance in China, Heilongjiang science, issue10,pp.48-49,2016.

[4] D.H.Hu, Markov processes in random environments. Beijing, Higher Education Press, 2011.

[5] D.X.Fan, Research on forecast method of railway passenger volume based on optimized grey markov chain model, ChongqingJiaotong University, PP.63, Apr.2015.

[6] F.X.Jiang,Y.X.Sun, The application of markov analysis in the passenger transport market. Technology \& Economy in Areas of Communications, issue 5, pp.100-102,2011.

[7] P.M.liao, Based on the Markov chain state transfer probability matrix, the commodity market state forecast.statistics and decision, issue 2,pp. 97-99,2015.

[8] S.Tao, Inventory strategy for luxury sales based on Markov chain, Scientific and Technological Innovation,pp.127,Nov.2016.

[9] A.N.Liang,Application of Markov Chain in Weather Forecast, Value engineering, issue8,pp.262-264,2016.

[10] F.L.Gao,M.H.Du,J.L.Sun, Application of $M$ arkov chain in $w$ eather forecast, Journal of Qiqihar University(Natural Science Edition), Vol.32,No.1 pp.90-92,Jan.2016.

[11] G.Zhi, method and application progress of prediction and early warning of infectious diseases (two).modern distance education of Chinese medicine in Chinese medicine, issue 16,pp.161163,2012 .

[12] W.H.Li,Y.T.Yang,N.H.Zeng,Application of Markov Chain in Prediction of Tuberculosis Morbidity in South Military, Military Medical Journal of South China,Vol.31,No.5,May.2017.

[13] F.L.Gao,X.Lin,M.H.Du, Analysis of the differences in mathematical ability between male and female college students based on Markov chain.Journal of Jianghan University(Natural Science Edition),pp.26-29,2016.

[14] Z.Chen,C.F.Lian,R.K.Pan, Application of the model of absorption Markov chain in the prediction of employment trend of agricultural transfer population in Shanxi province,economist,issue9,pp.151-231,2015.

[15] X.Q.Cai,Q.H.Song,X.P.Zu, Application of weighted markov chain in rice yield prediction, Journal of Science of Teachers' College and University,pp.28-30,Aug.2015 